\title{
Núcleo Especial do Programa Segundo Tempo na ESEF - UFPel
}

\section{Second Half Special Program in ESEF - UFPel}

Rev Bras Ativ Fis Saúde p. 236-241

DOI:

http://dx.doi.org/10.12820/rbafs.v.18n2p236

1 Escola Superior de Educação Física, Universidade Federal de Pelotas, Pelotas, RS

\author{
Angélica Xavier Kalinoski ${ }^{1}$ \\ Laura Garcia Jung ${ }^{1}$ \\ Gabriela Padilha Hax ${ }^{1}$ \\ Alexandre Carriconde Marques ${ }^{1}$ \\ Gabriela Brisolara Xavier ${ }^{1}$
}

\section{Resumo}

As desigualdades sociais e econômicas ainda colocam-se como um problema a ser superado no Brasil. Uma das soluções para amenizar esses problemas são os programas sociais que tem como finalidade oportunizar a igualdade e a inclusão social. Dentre alguns programas sociais, encontra-se o Programa Segundo Tempo (PST) desenvolvido pelo Ministério do Esporte (ME), com o objetivo de democratizar o acesso à prática esportiva dos alunos da educação básica e superior. Em sua nova remodelação, com intenção de atingir vários segmentos excluídos no contexto social, o PST desenvolveu núcleos especiais, entre eles um núcleo para jovens com deficiência. A partir de 2008 a UFPel, por intermédio da ESEF, formou uma parceria com a Secretaria Nacional de Esporte, Educação, Lazer e Inclusão Social do ME para o desenvolvimento de um núcleo Piloto Especial, visando atender preferencialmente crianças e jovens com deficiência com idades entre 12 e 21 anos. A partir de 2012, o projeto renovou a parceria por um ano, desta vez como Núcleo Especial e com as diretrizes já definidas. O trabalho é desenvolvido por professores e acadêmicos da ESEF/UFPel, no atendimento de 100 alunos com e sem deficiências. As atividades ocorrem três vezes por semanas por duas horas, sendo desenvolvidas atividades esportivas, atividades suplementares e atividades complementares de artes. Com essas oportunidades observa-se uma mudança no estilo de vida desses alunos. $\mathrm{Na}$ avaliação junto com as famílias e na observação dos monitores, percebe-se uma mudança de comportamento, com visível melhoria da autoestima, autoconfiança, independência e autonomia. Com a continuidade desse programa espera-se uma melhoria da qualidade de vida.

\section{Palavras-chave}

Programas sociais; Educação Física; Saúde; Inclusão.

\begin{abstract}
Economical and social disparities remain as a problem to overcome. One solution to reduce these problems are social programs that have the aim of provide equality and social inclusion. Among some social programs, is the Programa Segundo Tempo (PST) which was developed by the Sport Ministry (SM) with the goal of democratizing the access to sports practice to students from basic and higher education. In its new remodeling, with the intent to reach several segments excluded from the social context, the PST developed special centers, among them, one destined to disabled children. Since 2008, UFPel and ESEF, build a partnership with the National Department of Sport, Education, Leisure and Social Inclusion from SM to the development of a pilot special center, aiming to attend preferably children and youth with disabilities in ages ranging from 12 to 21 years old. In 2012, the project renewed the partnership for one year, but this time as a special center with already defined guidelines. The work is developed by teachers and academics from ESEF/UFPel, attending 100 students with and without disabilities. Activities take place three times a week for two hours, being promoted sports activities. With these opportunities is observed a change in this student's lifestyle. An evaluation conduced together with the families and the observation of monitors demonstrates change in behavior, with improvement in self-esteem, self-trust, independency and autonomy. With the continuation of this program improvement in quality of life is expected.
\end{abstract}

\section{Keywords}

Social Programs; Disability; Physical Education; Health; Inclusion. 


\section{INTRODUÇÃO}

É dever da família, da sociedade e do Estado assegurar à criança e ao adolescente, com absoluta prioridade, o direito à vida, à saúde, à alimentação, à educação, ao lazer, à profissionalização, à cultura, à dignidade, ao respeito, à liberdade e à convivência familiar e comunitária, além de colocá-los a salvo de toda forma de negligência, discriminação, exploração, violência, crueldade e opressão ${ }^{1}$. Infelizmente, no Brasil como em outros países, as desigualdades sociais e econômicas ainda colocam-se como um problema a ser superado ${ }^{2,3}$.

Uma das soluções para amenizar esses problemas são os programas sociais que tem como finalidade oportunizar a igualdade e a inclusão social ${ }^{4,5}$. Instituições, como as universidades, devem ser promotoras de programas sociais, visto que os mesmos, além de oportunizar novas oportunidades a comunidade também auxiliam na formação acadêmica. Assim, juntamente com a extensão e o ensino, está atrelado a pesquisa com as quais podem auxiliar para o melhoramento das diretrizes dos programas ofertados, qualificando assim o atendimento a comunidade.

Essa parceria com instituições torna-se necessária na medida que propiciará a atuação de profissionais qualificados fornecendo um melhor atendimento à sociedade, como é o caso das pessoas com deficiências que na maioria das vezes não estão inseridas na sociedade por falta de qualificação profissional. Percebe-se que a atual situação do Brasil, nesse contexto torna difícil a inclusão das pessoas com deficiência devido à falta de estrutura física, capacitação de profissionais, oportunidades e preconceito.

Dentre alguns programas sociais, encontra-se o Programa Segundo Tempo (PST) desenvolvido pelo Ministério do Esporte e que tem como objetivo democratizar o acesso e estimular a prática esportiva dos alunos da educação básica e superior através do esporte educacional em que se propõem a ensinar o esporte propriamente dito e a ensinar pelo esporte, através do desenvolvimento de competências, comportamentos, atitudes, valores (GRECO et. al, 2009) .

Com a evolução do programa e percepção dos avaliadores do Programa de que o número de pessoas com deficiência atendidas nos núcleos era baixa, foi elaborado o PST Especial, primeiramente como projeto piloto e agora com as diretrizes já definidas, visando atender crianças e jovens com deficiência e em situação de risco social. A contribuição desse estudo está em divulgar no meio científico as propostas que estão sendo realizadas para o aumento dos níveis de atividade física das pessoas com deficiência, formas de facilitar o processo inclusivo deles na sociedade, bem como servir de parâmetros para a realização de programas esportivos para essa população através do esporte educacional.

Uma das importâncias do PST Especial é oportunizar o convívio entre deficientes e pessoas sem deficiência entre ambos os sexos. Meninos e meninas, deficientes e não deficientes juntos, ocupando o mesmo espaço e praticando a mesma atividade esportiva podem, na convivência, aprender a relacionar-se com o outro, sobre como interagir com a diferença, em diferentes possibildades, aprender a compartilhar e resolver conflitos ${ }^{7}$. Dessa forma, todo o programa deve conter desafios a todos os alunos, permitir a participação de todos, respeitar suas limitações, promover autonomia e enfatizar o potencial no domínio motor. Assim, ocorrerá a participação de todos de acordo com seus limites e capacidades ${ }^{7,8,9,10}$.

Nesse contexto o programa tem como objetivo, democratizar o acesso ao esporte educacional de qualidade, como forma de inclusão social, contribuindo para a efetivação dos direitos e construção da cidadania das crianças e adolescentes com e sem deficiência. Quanto aos acadêmicos monitores participantes, o objetivo é capacitar o futuro profissio- 
nal da área da Educação Física, para desenvolver o ensino das atividades físicas para pessoas com deficiências, através da vivência de situações concretas de ensino-aprendizagem.

\section{FUNCIONAMENTO}

O projeto é desenvolvido pela Escola Superior de Educação Física da Universidade Federal de Pelotas - RS. O público alvo desse núcleo tem uma característica diferenciada, pois pretende estimular maior participação de crianças e jovens com deficiência. Estudos mostram que o nível de atividade física (AF) das pessoas com deficiência é inferior ao da população em geral ${ }^{11,12}$. Isso ocorre pelas limitações para a realização de atividades cotidianas associadas às deficiências.

Assim, o estímulo à prática de $\mathrm{AF}$ é importante porque as pessoas com deficiência têm necessidades de cuidado à saúde adicionais e mais complexos do que os da população em gera ${ }^{13}$ e a prática de atividades físicas é uma das variáveis determinantes na prevenção das doenças crônicas não transmissíveis. Além disso, no aspecto social, a atividade física e esportiva tem auxiliado pessoas com deficiência a não só adquirir autonomia e independência, como também resgatar sua autoestima, autoconfiança, as relações pessoais e seu equilíbrio emocional ${ }^{14}$.

O projeto não pretende ser um núcleo somente de alunos com deficiência, pois dessa forma estaria indo contra todo o processo de inclusão atual. Optou-se em trabalhar com crianças e jovens com déficit intelectual e em situação de risco. Foram definidas como pessoas em situação de risco aquelas que na sua vida se enquadram em pelo menos uma destas situações: possuem uma família desagregada; sofrem maus tratos e abusos no âmbito familiar ou vivem em locais sem condições de saneamento básico. Foram adotados como critérios de seleção os seguintes requisitos: disponibilidade de horário, ter déficit intelectual, situação de risco social, menor renda familiar e dificuldade de aprendizado.

Para a divulgação do programa foi realizado um contato inicial com escolas especiais e regulares da cidade de Pelotas, a fim de explicar a proposta do programa. Realizou-se também uma divulgação na mídia por meio de rádio, jornal e televisão para convocação de reunião com pais e responsáveis. A partir da divulgação e de acordo com as definições do Programa Segundo Tempo, para o projeto piloto foram selecionadas 100 crianças e adolescentes entre 12 e 21 anos, de ambos os sexos. Após as definições das diretrizes, a faixa etária de atendimento ficou entre 07 e 21 anos. Desses participantes, $70 \%$ têm déficit intelectual e os demais estão em situação de risco social. Cada aluno é atendido durante seis horas na semana, divididas em três dias de atendimento. Para a realização da inscrição dos alunos menores de 18 anos ou dos que possuem deficiência, a presença dos pais é indispensável para estarem cientes de que o filho participará do Programa, bem como dos horários das atividades.

Para atender essas crianças e jovens, o programa conta com um coordenador geral, um coordenador de núcleo, cinco monitores (quatro para atividades esportivas e físicas e um de atividade complementar). Os monitores foram qualificados através da capacitação pedagógica oferecida pelo Ministério do Esporte para melhor atender as demandas do projeto em relação às atividades.

O projeto visa desenvolver atividades esportivas de futebol, basquete, basquete em cadeiras de rodas, vôlei, handebol, rugby, bocha, golbol, atletismo e natação. Para que essas atividades fossem realizadas durante os 24 meses do projeto piloto, os trabalhos foram feitos em forma de rodízio bimestral, ou seja, a cada bimestre um esporte coletivo era desenvolvido, para dessa forma oportunizar a prática de diversos esportes.

Nas atividades suplementares foram trabalhadas sessões para a reeducação mo- 
tora (equilibrio, coordenação, ritmo, agilidade, mobilidade e esquema corporal); dança, ginástica rítmica, exercícios físicos com bolas bobath, atividades cênicas, xadrez e palestras informativas (saúde, nutrição, sexualidade e outros temas solicitados). O projeto oportuniza também a prática de artes, como atividade complementar.

Foram feitas observações diretas e conversas com os pais para a obtenção dos resultados que os beneficiados obtiveram ao longo das atividades realizadas no programa. Percebeu-se que a participação no programa permitiu o desenvolvimento da aptidão física relacionada à saúde e o desempenho motor. Em relação ao aspecto social, observou-se juntamente com os pais/responsáveis que o programa oportuniza situações de controle de estresse, ansiedades e frustrações, diminuição do preconceito, motivação para a vida, melhoria da autoestima e autoimagem. Também de acordo com relato dos pais, melhorou o nível de independência e autonomia, relacionamento interpessoal/amizades, quebra de rotina e o desenvolvimento de atitudes positivas e melhores níveis de independência e autonomia na realização das tarefas de vida diária. Além disso, o projeto propicia um conhecimento da comunidade e das famílias sobre a importância da atividade esportiva.

Para a avaliação dessas atividades e o controle dos resultados, foi realizado um controle de frequência em todas as atividades desenvolvidas. Entre elas, foram realizadas avaliações da aptidão física proposta pelo PROESP-BR ${ }^{11}$ e acompanhamento dos níveis de crescimento (massa corporal e estatura). Para avaliação qualitativa das aulas, os monitores faziam relatórios semanais sobre o rendimento dos alunos durante as atividades propostas.

Para avaliar a qualidade do projeto, houve acompanhamento através de reuniões, diagnóstico de participação, elaboração de relatório do projeto contendo frequência, avaliação do material, elaboração de relatório sobre atuação de monitores e coordenadores e registro fotográfico das ações desenvolvidas.

A eficácia do projeto deu-se a partir dos dados relatados do projeto piloto, sendo que suas configurações foram firmadas para definir as diretrizes do Programa Segundo Tempo Especial. A partir da eficácia do piloto em 2011 foram determinadas as diretrizes para esse tipo de núcleo. A eficiência do programa pode ser percebida depois que o Ministério do Esporte lançou um edital para que todas as universidades pudessem implementar este tipo de núcleo. Então, em 2012, a Universidade Federal de Pelotas implementou o núcleo com as diretrizes já estabelecidas, dando continuidade ao projeto na ESEF/UFPel.

\section{O MODELO LÓGICO DO PROGRAMA SEGUNDO TEMPO ESPECIAL}

Os recursos financeiros do programa são provenientes do Ministério do Esporte em forma de materiais didáticos, reforço alimentar e de bolsas para os recursos humanos (coordenadores e monitores). Para completar os recursos, a infraestrutura utilizada foi a da ESEF-UFPel.

Durante as atividades desenvolvidas pelo núcleo foram realizadas, mensalmente, reuniões com pais e alunos, com intuito de coletar informações sobre o desenvolvimento das atividades, permitindo identificar como o PST contribui para o desenvolvimento de crianças e adolescentes. Ao final de cada bimestre, de posse das informações coletadas, as estratégias de ensino foram modificadas, quando necessário, com vistas a ampliar o desenvolvimento integral das crianças e adolescentes.

Foram feitas avaliações, acompanhamento e análises das variáveis antropométricas, da aptidão física, do estado nutricional e dos hábitos de vida dos participantes. 


\begin{tabular}{|c|c|c|c|c|c|}
\hline Insumos & Atividades & Produtos & Resultados iniciais & $\begin{array}{l}\text { Resultados } \\
\text { intermediários }\end{array}$ & $\begin{array}{l}\text { Resultados a longo } \\
\text { prazo }\end{array}$ \\
\hline \multirow[t]{2}{*}{$\begin{array}{l}\text { RECURSOS } \\
\text { HUMANOS } \\
\text { - } 01 \text { Coordenador } \\
\text { Geral } \\
\text { - } 01 \text { Coordenador de } \\
\text { Núcleo (Profissional } \\
\text { de Educação Física) } \\
\text { - } 04 \text { monitores } \\
\text { (alunos } \\
\text { - de graduação em } \\
\text { - Educação Física) } \\
\text { INFRAESTRUTURA } \\
\text { - Escola Superior de } \\
\text { Educação Física - } \\
\text { UFPel } \\
\text { - RECURSOS } \\
\text { FINANCEIROS } \\
\text { - Ministério do } \\
\text { Esporte }\end{array}$} & $\begin{array}{l}\text { - Prática de esportes } \\
\text { coletivos e individuais } \\
\text { - Atividades } \\
\text { complementares de } \\
\text { Artes } \\
\text { - Atividades } \\
\text { suplementaresde } \\
\text { reeducação motora } \\
\text { - Palestras informativas } \\
\text { sobre aspectos } \\
\text { relacionados à saúde } \\
\text { - Passeios educacionais } \\
\text { - Festas temáticas } \\
\text { - Avaliação física } \\
\text { através do PROESP } \\
\text { - Acompanhamento } \\
\text { dos níveisde } \\
\text { crescimento (estatura } \\
\text { e massa corporal) } \\
\text { - Controle de } \\
\text { frequência } \\
\text { - Reuniões com os pais }\end{array}$ & $\begin{array}{l}\text { - } 100 \text { crianças e } \\
\text { jovens praticando } \\
\text { esporte com } \\
\text { qualidade } \\
\text { - Complementação } \\
\text { na formação dos } \\
\text { graduandos } \\
\text { - Inclusão dos } \\
\text { alunos e } \\
\text { responsáveis na } \\
\text { comunidade }\end{array}$ & $\begin{array}{l}\text { - Aumento do nível } \\
\text { de prática de } \\
\text { atividade física dos } \\
\text { beneficiados } \\
\text { - Aumento do nível de } \\
\text { conhecimento dos } \\
\text { alunos e responsáveis } \\
\text { sobre saúde } \\
\text { - Diminuição o tempo } \\
\text { em comportamento } \\
\text { sedentário } \\
\text { - Melhora das } \\
\text { habilidades motoras } \\
\text { dos beneficiados } \\
\text { - Socialização entre os } \\
\text { alunos } \\
\text { - e com os monitores } \\
\text { - Quebra de rotina } \\
\text { - Relacionamento } \\
\text { interpessoal/amizades } \\
\text { - Adesão ao Programa }\end{array}$ & $\begin{array}{l}\text { - Incorporação } \\
\text { de um estilo } \\
\text { de vida ativo } \\
\text { - Prevenção e } \\
\text { controle da } \\
\text { obesidade } \\
\text { e fatores } \\
\text { associados } \\
\text { - Aumento } \\
\text { do nível de } \\
\text { atividade física } \\
\text { no tempo livre } \\
\text { - Vivência de } \\
\text { modalidades } \\
\text { esportivas }\end{array}$ & $\begin{array}{l}\text { - Melhora dos } \\
\text { níveis de } \\
\text { atividade física } \\
\text { - Inclusão dos } \\
\text { beneficiados na } \\
\text { comunidade } \\
\text { - Melhora } \\
\text { naqualidade de } \\
\text { vida } \\
\text { - Melhora dos } \\
\text { níveis de } \\
\text { aptidão física } \\
\text { - Recursos } \\
\text { humanos } \\
\text { melhor } \\
\text { qualificados } \\
\text { - Definição das } \\
\text { diretrizes para } \\
\text { o Programa } \\
\text { Segundo Tempo } \\
\text { Especial }\end{array}$ \\
\hline & $\begin{array}{l}\text { Capacitação } \\
\text { - Capacitação } \\
\text { pedagógica oferecida } \\
\text { pelo Ministério } \\
\text { do Esporte aos } \\
\text { coordenadores } \\
\text { - Monitores capacitados } \\
\text { pelo Coordenador de } \\
\text { Núcleo }\end{array}$ & & & & \\
\hline
\end{tabular}

Também foram oferecidas palestras informativas, festas temáticas e passeios educacionais que permitiram o desenvolvimento integral das crianças e adolescentes.

Simultaneamente o Programa teve como produto direto o atendimento a 100 crianças e jovens além de seus pais/responsáveis. Soma-se aos produtos o número de participantes que desistiram durante o desenvolvimento do Programa e, em razão disso, essas vagas foram disponibilizadas a outras pessoas.

Espera-se que com esse programa as pessoas atendidas tenham aumentado os níveis de prática de atividade física, melhora da aptidão física, melhoria na integração social e, consequentemente, uma melhora, na sua qualidade de vida.

\section{CONCLUSÃO}

A partir do desenvolvimento das atividades percebeu-se melhoria da qualidade de vida dos beneficiários através da mudança no estilo de vida dos alunos. No momento em que passaram a participar de um programa esportivo modificaram sua rotina, diminuindo inclusive comportamento sedentário, pois passaram a ter que se deslocar até o local de atendimento, também diminuindo o tempo ocioso em casa. Outro aspecto é o envolvimento social, enquanto ficavam apenas em casa não tinham interação social, tanto os alunos quantos os pais. 
A participação da família foi importante no contexto do programa, pois a melhoria dos níveis de conhecimento facilitou suas relações com as necessidades de atendimento de seus filhos, e na sua relação com a comunidade em geral. Outro ponto importante diz respeito a melhoria da aptidão física e a coordenação motora observadas durante as aulas, onde inicialmente havia alunos que não conseguiam desenvolver determinadas atividades e que, com o andamento do projeto, esses alunos passaram a realizar as atividades que tinham dificuldades.

Dessa forma, a realização do projeto piloto permitiu a definição das diretrizes para o Programa Segundo Tempo Especial, oportunizando assim, que essa população seja atendida em outros núcleos, usufruindo dos mesmos benefícios. Consequentemente, ocasionará um aumento do número de pessoas com deficiência participando de programas sociais e, neste contexto, um projeto voltado para atividades de esporte educacional.

\section{REFERÊNCIAS}

1. http://www.planalto.gov.br/ccivil_03/constituicao/constitui\%C3\%A7ao.htm. Acessado em 01 de outubro de 2011.

2. Gaya A. Programa Segundo Tempo - Introdução. In: Fundamentos pedagógicos para o Programa Segundo Tempo. Maringá: EDUEM, 2008: 09-12.

3. Gomes MA, Pereira, MLD. Família em situação de vulnerabilidade social: uma questão de políticas públicas. Ciênc. Saúde Coletiva. 2005; 10: 357-363.

4. Vieira E. A política e as Bases do Direito Educacional. Cad. CEDES. 2001; 21: 9-29.

5. Inclusão: Revista da Educação Especial. Secretaria de Educação Especial. Brasilia. 2005; 1: 01-56.

6. Greco PJ, Silva AS, Santos LR. Organização e Desenvolvimento Pedagógico do Esporte no Programa Segundo Tempo. In: Fundamentos pedagógicos para o Programa Segundo Tempo: da reflexão à prática. Mariangá: Eduem, 2009: 163-206.

7. Cidade RE, Busto RM. Inclusão, Gênero e Deficiência. In: Fundamentos Pedagógicos para o Programa Segundo Tempo. Maringá: EDUEM, 2008: 123-138.

8. Pedrinelli VJ. Educação Física Adaptada: Conceituação e Terminologia. In: Educação Física e Desporto para Pessoas Portadoras de Deficiência. Brasîlia: MEC-SEDES, SESI-DN, 1994: 7-10.

9. Jung LG. Inclusão de crianças e jovens com deficiência nas aulas de Educação Física. 2010.54f. Trabalho de Conclusão de Curso. Curso de Licenciatura em Educação Física. Universidade Federal de Pelotas, Pelotas.

10. Amaral LA. Pensar a Diferença/Deficiência. Brasília: Coordenadoria Nacional para Integração da Pessoa Portadora de Deficiência, 1994.

11. Finlayson J, Jackson A, Cooper SA, Morrison J, Melville C, Smiley E, Allan L, Mantry D. Understanding Predictors of Low Physical Activity in Adults with Intellectual Disabilities. Journal of Applied Research in Intellectual Disabilities, 22, 236-247, 2009.

12. Rimmer JH, Marques AC. Physical activity for people with disabilities. The Lancet. v 380, p 193-195, 2012.

13. Cooper SA, Morrison J, Melville C, Finlayson J, Allan L, Martin G. et al. Improving the health of people with intellectual disabilities: Outcomes of a health screening programme after 1 year. Journal of Intellectual Disability Research, v 50, pp. 667-677, 2006.

14. Oliveira AAB, Perim GL. Fundamentos pedagógicos do programa segundo tempo: da reflexão a prática. UEM, 2009.

15. http://www.proesp.ufrgs.br. Acessado em 20 de março de 2012.

$$
\begin{array}{r}
\text { Endereço para Correspondência } \\
\text { Angélica Xavier Kalinoski } \\
\text { Rua Gonçalves Chaves, } 824 \text { aparta- } \\
\text { mento } 303 \\
\text { CEP: } 96015-560 \\
\text { E-mail: angelica.kalinoski@gmail.com } \\
\text { Telefone: } 5198760207
\end{array}
$$

Recebido 14/08/2012

Revisado 25/03/2013

Aprovado 14/06/2013 